\title{
The Progress of Patients with Blunt Abdominal Trauma Depending on the Number of the Damaged Organs.
}

\author{
Agron Dogjani ${ }^{1 *}$, Kastriot Haxhirexha ${ }^{2}$, Arben Gjata ${ }^{1}$, Amarildo Blloshmi ${ }^{3}$, Hysni Bendo ${ }^{4}$
}

Received: 04 October 2021 / Accepted: 25 October 2021 / Published online: 20 January 2022

This article is published with open access at https://journal.astes.org.al

(C) The author(s) 2022. \& Copyright (C) 2022, the Albanian Society for Trauma and Emergency Surgery

(c) The Albanian Journal of Trauma and Emergency Surgery is an Open Access Journal. All articles are distributed under the terms of the Creative Commons Attribution Non-Commercial License: http://creativecommons.org/licenses/by-nc/4.0/) which permits unrestricted non-commercial use, distribution, and reproduction in any medium provided the original work is properly cited.

\begin{abstract}
Introduction: Blunt abdominal trauma (BAT) is one of the most common traumatic emergencies and is associated with significant morbidity and mortality, which is associated with a number of causes despite improvements in their management. This study aims to assess 308 cases of BAT and their management depending on the number of Injured organs.

Material and Methods: The study has a retrospective character and it has been realized within the period of time from January 20016 to December 2018. In our study are included 308 trauma cases admitted at the University Hospital of Trauma in Tirana Albania. All recorded data were grouped as follows; Demographic data, trauma mechanism, admission time in hospital, the mode of management...

Results: The sample of the population was chosen by chance which means that this study is included all trauma cases presented at the emergency department and hadn't any study limitation.

In our study, based on the mechanism of trauma, motor vehicle accident [191(62\%)] was the most common mechanism of injury, and most of the patients in our study were male [231(75\%)], female [77 (25\%)] with an M:F ratio of 3:1. The spleen [118 (38.3\%)] was the most damaged organ after BAT, and the most common surgical procedure performed was splenectomy [53 (35.5\%)].

The majority of traumatized patients presented to the Emergency Department in stable hemodynamic condition [145 (47.1\%)]

Conclusions: The progress of patients with BAT has a close relationship with The number of injured organs. The number and presence of EAI organs were important which worsen patient conditions and influence at the prognosis. The time of hospitalization of patients has a lot to do with the result. Early diagnosis and prompt treatment can save many lives.

Keywords: Blunt abdominal trauma, Injured organs, early diagnosis, resuscitation
\end{abstract}

\begin{abstract}
Abbreviations:
Blunt abdominal trauma - BAT; Focused Assessment with Sonography in Trauma - FAST; Computed Tomography - CT; Operative Management - OM; Non-Operative Management - NOM: Instable Hemodynamically - InStHd: Stable Hemodynamically - StHd; Motor Vehicle Accidents -
\end{abstract}

Original article, no submission or publication in advance or in parallel

* Corresponding author:

Asc. Prof. Dr.Agron Dogjani, MD, Ph.D. FACS, FICS, FISS

$\triangle$ agrondogjani@yahoo.com

1 University of Medicine of Tirana, ALBANIA

2 General Surgeon at Clinical Hospital of Tetovo, RN of MACEDONIA.

3 General Physician National Centre of Medical Emergency, Tirana, ALBANIA

4 Resident in Internal Medicine at the SRH Clinic, Germany
MVA; Falling from Height - FfH; Hemodynamic Conditions - HdCo; Vulnerable Hemodynamicaly - VuHd; ExtraAbdominal Injuries - EAI; Multiple Organ Failure $M O F$;

\section{Introduction}

Trauma has been called the neglected disease of modern society. Trauma is the leading cause of death and disability in developing countries and the most common cause of death under 45 years of age.[1]

World over injury is the 7 th cause of mortality and abdomen is the third most common injured organ. Abdominal injuries require surgery in about $25 \%$ of cases. $85 \%$ of abdominal traumas are of blunt character.[3]

The spleen and liver are the most commonly injured organs as a result of blunt trauma. Clinical examination alone is inadequate because patients may have altered mental status and distracting injuriries. Initial resuscitation 
along with and abdomen are very beneficial to detect those patients with minimal and clinically undetectable signs of abdominal injury and are the part of recent management guidelines.

Approach to trauma should be systemic and prioritized. About $10 \%$ of patients have persistent hypovolemic shock as a result of continuous blood loss in spite of aggressive fluid resuscitation and require an urgent laparotomy.

Damage control laparotomy is a life saving procedure for such patients with life-threatening injuries and to control haemorrhage and sepsis.[17] On the other spectrum, there has been increasing trend towards non operative management (NOM) of blunt trauma amounting to $80 \%$ of the cases with failure rates of 2-3\%.[4]

NOM is a standard protocol for hemodynamically stable solid organ injuries. Pre-hospital transportation, initial assessment, thorough resuscitative measures and correct diagnosis are of utmost importance in trauma management.[2]

\section{Materials and Methods}

The study has a retrospective character and it has been realized within the period of time from January 20016 to December 2018. In our study are included 308 trauma cases admitting at University Hospital of Trauma in Tirana, Albania.

All the trauma patient after Primary Survey \& resuscitation, detailed clinical history, physical examination, laboratory tests and x-rays, with focused assessment with sonography in trauma (FAST) was done to arrive at the diagnosis. CT scanner was done in most of the cases.

Patients were categorized in two groups instable and stable hemodynamically (InStHd and StHd)

Patient management progress was monitored step by step, and the decision on treatment (NOM vs OM) was based on algorithms already all previously accepted.

Patients who failed NOM and were InStHd, continued to worsen despite proper resuscitation or who had evidence of involvement of cavitary organs were taken for immediate laparotomy.

Other variables such as age, gender, cause of BAT, time of presentation of the patient to the emergency department, signs and clinical symptoms, finding in the operating table.

The various procedures were very important in the progress of traumatized patients, the performed surgery, associated extra-abdominal injuries, postoperative complications...

\section{Results}

There was included in this study 308 patients, male 231 $(75 \%)$, female $77(25 \%)$ of cases. The ranging of patients age was; a $-<14$ years old 68 (22\%), b - 14-40 years old 117 $(37.9 \%)$; c - over 40 years old $123(40.1 \%)$ of cases. (Tab 1).
Table 1: Age and gender-wise distribution

\begin{tabular}{|l|l|l|l|}
\hline Parameter & & No of patients & \% of patients \\
\hline Age group & Less than 14 & 68 & 22 \\
(years) & $14-40$ years old & 117 & 37.9 \\
\cline { 2 - 4 } & over 40 years & 123 & 40.1 \\
\hline \multirow{2}{*}{ Gender } & Males & 231 & 75 \\
\cline { 2 - 4 } & Females & 77 & 25 \\
\hline
\end{tabular}

The Epidemiological data based on mechanism of injuries were; a - MVA 191 (62\%); b- Falling from height (FfH) - 86 (28\%); c - Assault - 31 (10\%) of all cases. Motor vehicle accidents involving both pedestrians and vehicular accidents accounted for $62 \%$ majority of injuries (Tab 2).

\section{Table 2: Mechanism of Injury distribution}

\begin{tabular}{|l|c|c|}
\hline Mechanism of Injury & No of patients & \% of patients \\
\hline Motor vehicle accidents & 191 & 62 \\
\hline Falling from height & 86 & 28 \\
\hline Assault & 31 & 10 \\
\hline
\end{tabular}

Distribution in according to the mode of transportation to the hospital were; Privat Car 166 (53,9\%); Autoambulance 135 (43,8\%); Helicopter 7 (2,3\%) of cases;

The admission time in hospital was as followings: a - Immediately $246(79.8 \%)$; b - after 6 hours 37(12,1\%); c - after 24 hours $25(8,1 \%)$ of cases.

The Hemodynamic conditions ( $\mathrm{HdCo}$ ) in admission were as fellow: stable hemodynamicaly (StHd) in 145 $(47,1 \%)$, vulnerable hemodynamicaly (VuHd) in 68 $(22,1 \%)$; instable hemodynamicaly (InStHd) in 95(30.8\%) of cases. (Tab 3).

\section{Table 3: The distribution of the patient's data in admission of $D E$}

\begin{tabular}{|l|c|c|}
\hline The patient's data & No of patients & \% of patients \\
\hline The mode of transportation & & \\
\hline • Privat Car & 166 & $53,9 \%$ \\
\hline - Auto-ambulance & 135 & $43,8 \%$ \\
\hline • Helicopter & 7 & $2,3 \%$ \\
\hline The admission time in hospital & & \\
\hline a - Immediately & 246 & $79.8 \%$ \\
\hline b- after 6 hours & 37 & $12,1 \%$ \\
\hline c - after 24 hours & 25 & $8,1 \%$ \\
\hline The Hemodynamic conditions & & \\
\hline • stable hemodynamically & 145 & $47,1 \%$ \\
\hline • vulnerable hemodynamically & 68 & $22,1 \%$ \\
\hline • instable hemodynamicaly & 95 & $30.8 \%$ \\
\hline
\end{tabular}

The distribution of the initial clinical assessment for all recorded patients in the admission, in our study was as follow; the abdominal pain 209 (67.8\%); The abdominal tenderness $200(65 \%)$ of the cases; Hypovolemia was present in $95(30.8 \%)$ of the cases; the abdominal guarding 
in $72(23.3 \%)$ of the cases; Gastrointestinal haemorrhage $25(8.1 \%)$ of the cases; vomiting $240(78 \%)$ of the cases: Dyspnoea in 117 (38\%) of the cases; and haematuria in 38 $(12.3 \%)$ of the patients (Table 4$)$.

\section{Table 4: The distribution of the initial clinical assessment of the patients}

\begin{tabular}{|l|c|c|}
\hline The clinical assessment & No of patients & \% of patients \\
\hline The Abdominal Pain & 209 & 67.8 \\
\hline The abdominal tenderness & 200 & 65 \\
\hline Gastrointestinal haemorrhage & 25 & 8.1 \\
\hline Hypovolemia & 95 & 30.8 \\
\hline The abdominal guarding & 72 & 23.3 \\
\hline Nausea \&Vomiting & 240 & 78 \\
\hline Dyspnoea & 117 & 38 \\
\hline Haematuria & 38 & 12.3 \\
\hline
\end{tabular}

However, large amounts of blood can accumulate in the peritoneal and pelvic cavities without any significant or early changes in the physical examination findings. Bradycardia may indicate the presence of free intraperitoneal blood.

\begin{tabular}{|l|l|lc|}
\hline Correlations & & \multicolumn{2}{|c|}{ TRANSPORT mode } \\
\hline Kendall's tau-b & $\begin{array}{l}\text { Hemodynamic } \\
\text { conditionsin } \\
\text { Admission }\end{array}$ & $\begin{array}{l}\text { Correlation } \\
\text { Coefficient }\end{array}$ & 2.12 \\
\hline & Sig.(2-tailed) & & 0.48 \\
\hline & No & & 308 \\
\hline
\end{tabular}

Figure 1 Hemodynamic conditions in Admission and mode of transportation correlation

Through correlation coeficient of Kendal's tau-b, we can see that there is a relation between the Hemodynamic conditions in Admission and mode of transportation (Fig. 1)

We had also distribution of extra-abdominal injuries (EAI) in our study as follow; retroperitoneal hematoma $42(13.6 \%)$, Head Trauma $74(24 \%)$, and haemothorax 61 (20\%), and pneumothorax $26(8.4 \%)$ of the cases.

Associated injuries in our study were mainly rib fractures in $88(28.5 \%)$, Pelvic fractures $26(8.4 \%)$, Extremity fractures $48(16.6 \%)$ of the cases; spine fracture $18(5.8 \%)$ of the cases (Tab 5).

Table 5: The distribution of the extra-abdominal injuries

\begin{tabular}{|l|c|c|}
\hline Extra-Abdominal Injuries & No of patients & \% of patients \\
\hline The retroperitoneal hematoma & 42 & 13.6 \\
\hline Head Trauma & 74 & 24 \\
\hline Haemothorax & 61 & 20 \\
\hline Pneumothorax & 26 & 8.4 \\
\hline The Associated injuries & & \\
\hline The Rib Fractures & 88 & 28.5 \\
\hline The Pelvic fractures & 26 & 8.4 \\
\hline The Extremity fractures & 48 & 16.6 \\
\hline The Spine Fracture & 18 & 5.8 \\
\hline
\end{tabular}

Most of the accompanying injuries were NOM, while haemothorax and pneumothorax required chest drainage. To identify abdominal injuries, we used radiological examination such as: X-ray of the abdomen, FAST and CT scan of the abdomen and pelvis and multiple injuries were detected.

Based on the way of treatment there were NOM in 159 $(51.6 \%)$ of the cases, and with OM in 149 (48.4\%) of the cases. (Tab 6).

The time of surgery was a - within 1 - 2 hours 22 (14.7 $\%)$; b - after 2-6 hours 50 (33.5\%), c - after 6 - 24 hours 47 $(31.5 \%)$; d - after 24 hours $30(20.3 \%)$ of cases.

In according to the distribution of intra abdominal injured (IAI) organs including OC \& OS resulted these data; Lien 118 (38.3\%), Liver 98 (31.8\%), Kidney 33(10.7 \%), Pancreas 9 (2.9\%) Intestine 55(17.8\%), Urinary Bladder 19 (6.1\%), Urethra $13(3.2 \%)$, Mesentery tear 11 (3.5\%), Diaphragmal rupture $18(5.9 \%)$, Big vessels $6(1.9 \%)$, Retroperitoneal hematoma $116(37.6 \%)$ of the cases. (Tab 6).

Table 6: The distribution of the time of surgery and the organ injured

\begin{tabular}{|l|c|c|}
\hline The time of surgery & No of patients & \% of patients \\
\hline within 1- 2 hours & 22 & 14.7 \\
\hline after 2-6 hours & 50 & 33.5 \\
\hline after 6 - 24 hours & 47 & 31.5 \\
\hline after 24 hours & 30 & 20.3 \\
\hline Mode of Treatment & & \\
\hline - NOM & 159 & 51.6 \\
\hline - OM & 149 & 48.4 \\
\hline The Organ Injured & & \\
\hline Lien & 118 & 38.3 \\
\hline Liver & 98 & 31.8 \\
\hline Kidney & 33 & 10.7 \\
\hline Pancreas & 9 & 2.9 \\
\hline Intestine & 55 & 17.8 \\
\hline Urinary Bladder & 19 & 6.1 \\
\hline Urethra & 13 & 3.2 \\
\hline Mesentery tear & 11 & 3.5 \\
\hline Diaphragm rupture & 18 & 5.9 \\
\hline Big vessels & 6 & 1.9 \\
\hline Retroperitoneal hematoma & 116 & 37.6 \\
\hline
\end{tabular}

Based on the total cases is reported $159(51.6 \%)$ cases were with NOM and Splenic injury was in $65(40.8 \%)$, liver injured $46(28.9 \%)$; Pancreas trauma in $7(4.4 \%)$; renal injuries in 23 (14.4\%); Urinary Bladder contusion in 9 $(6.9 \%)$, Urethral injured $9(6.9 \%)$ of cases.

Based on the total cases is reported 149 (48.4\%) cases were with $\mathrm{OM}$ and the kind of surgery performed was as following: Splenectomy 53 (35.5\%), 52 Liver surgeries including: a) Liver Suture $34(22.8 \%)$, b) Perihepatic packing $12(8 \%)$, c) Selective vasal liver ligature 4 (2.6\%): 
d) Anatomical and atypical liver resections 2 (1.3\%); Nephrectomy - $8(5.3 \%)$, Kidney suture in $2(1.2 \%)$ : Pancreas repair 2(1.2\%): Intestine suture / resection 18 (12.1\%), Urinary Bladder Suture 10 (6.7\%), Urethra $4(1.2 \%)$ of cases.

The distribution of BAT patient data by age and number of damaged organs was as follows:

a - with one injured organ $203(65.9 \%)$; b- with two injured organs $61(19.8 \%)$; c - with more than two injured organs $44(14.2 \%)$ of cases.

The data on traumatized patients according to the number of damaged organs in relation to age, was as follows: 1 - with one injured organ in $229(74,3 \%)$ of cases; a) less than 14 years old - 43 (14\%); b) $14-40$ years-old - $98(32 \%)$; c) over 40 years - $88(28.6 \%) ; 2$ - with two injured organs in $55(17.8 \%)$ of cases; a) less than 14 years old - $8(2.6 \%)$; b) $14-40$ years-old - $19(5.2 \%)$; c) over 40 years - $28(9.1 \%)$; 3- with more than two injured organs $24(7.9 \%)$ of cases. a) less than 14 years old - $4(1,4 \%)$; b) $14-40$ years-old - $8(2.6 \%)$ of cases; c) over 40 years -12 (3.9\%) of cases. (fig. 2)

The length of hospital stay always depends on the number and degree of damaged organs, hemodynamic status on admission and ongoing, the presence of EAI, age and comorbidity of patients.

The type of Blunt abdominal trauma which is isolated or combined, where the outcomes of the isolated BAT were often more favourable.

The time of performing the Surgery which in cases with obvious injuries, or diagnosed in time, the sooner the $\mathrm{OM}$ is performed the better the results will be.
In general, the performance of patients with BAT, the impact of the number of damaged organs, it is quite complex and intertwined with a series of factors which make it difficult to accurately predict.

The number or presence of damaged extra-abdominal organs which aggravate the condition of the patient and consequently his progress, but often extra-abdominal injuries result in intra-abdominal injuries such as. Rib fractures.

Mortality rate in our study was seen in $6(2 \%)$ cases in our study out of which 2 was intra-operative. Commonest cause was irreversible shock in $2(0.7 \%)$ followed by cardiopulmonary arrest $2(0.7 \%)$, and in $2(0.7 \%)$ reason Multiple Organ Failure (MOF).

Post-operative complications most frequently observed in our study were wound infection in $8(2.6 \%)$ and wound dehiscence in $5(1.6 \%)$ of cases.

\section{Discussion}

BAT is an arduous task even to the best of traumatologists. Injuries ranging from single organ to mutilating multi organ trauma may be produced by blunt abdominal trauma.

Abdominal findings may be absent in $40 \%$ of patients with hemoperitoneum. Sometimes, clinical evaluation of blunt abdominal injuries may be masked by other more obvious external injuries. $[2,4]$

Non therapeutic laparotomies have significantly reduced with proper and timely applications of imaging methods in BAT patients along with physical examination. Unrecognized abdominal injury is a frequent cause of preventable death after trauma. $[5,6]$

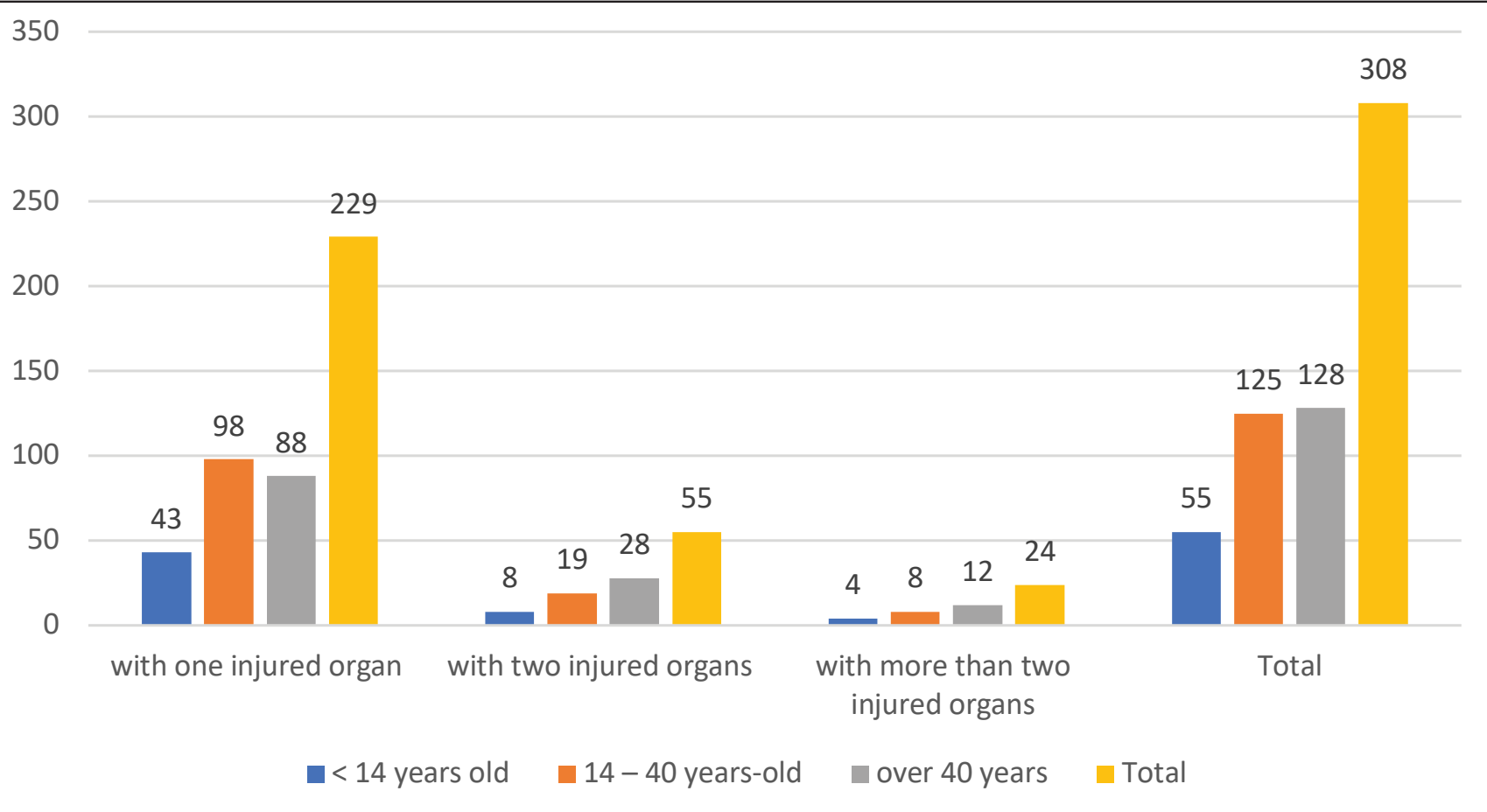

Figure 2 The data on traumatized patients according to the number of damaged organs in relation to age 
The patients who had sustained blunt abdominal trauma may have sustained injurysimultaneously to other systems and it is particularly important to examine for injuries of head, thorax and extremities. Vigilance and care of injuries in any of these systems may take precedence over abdominal trauma.[2]

Out of 308 cases in our study $78 \%$ of patients were in over 14 years old of age group. This goes in accord with studies of Davis et al. [8] and Lowe et al. [9] 79\% cases were males and $21 \%$ were females with an M:F ratio of 3.7:1.

The male preponderance in our study reflects that the greater mobility of males for either work, such as drivers and mechanics for automobiles or recreational activities may be resulting in a higher exposure to the risk of traffic injuries.[17]

MVA in $191(62 \%)$ of cases, is the leading cause of BAT in our study

This was equivocal with other studies conducted by Perry et al. [10] and Morton et al. [11] Thus prevention of accidents can decrease fatality. Commonest intra-abdominal injury was splenic injury with $118(38.3 \%)$ followed by liver injury in $98(31.8 \%)$ of cases.

Th commonest hollow organ injury was small bowel perforation in 55(17.8\%), and most common bowel injured was ileum in our study.

These results were consistent with other studies of Davis et al. [8] and Morton et al. [11]

In blunt trauma surgeon's main concern is control of haemorrhage, but how it can be best done with safety and less morbidity, depends on grade, severity and site of injury. [17]

Procedures done for splenic trauma in our study were splenectomy in $53(35.5 \%)$, and Splenectomy was done for most of grade 4 and 5 trauma and hemodynamically unstable patients of lesser grades.

In our Study we have performed in 52 cases Liver surgeries including: a) Liver Suture 34 (22.8\%), b) Perihepatic packing 12(8\%), c) Selective vasal liver ligature $4(2.6 \%)$ : d) Anatomical and atypical liver resections 2 $(1.3 \%)$;

Hemodynamically stable patients were followed with series physical examinations; ultrasonography or CT scans thus, avoiding unnecessary laparotomy.

Kidney and urinary bladder injuries were $33(10.7 \%)$ of cases, frequently associated with pelvic fractures $26(8.4 \%)$ of cases.

Nephrectomy through transperitoneal approach was done in $8(5.3 \%)$, cases of extensive renal lacerated Grade 5 injury and the patient recovered uneventfully, and Kidney suture in $2(1.2 \%)$ of cases.

All patients of renal trauma who were NOM were followed with regular CT scans and all performed well in their course. Most grade I-IV renal injuries can be managed non-operatively. [7, 8] The absolute indications for surgery include renal pedicle injury, shattered kidney, expanding hematoma, and hemodynamic instability.
In patients with intraperitoneal urinary bladder injury was performed $u$. bladder suture in $10(6.7 \%)$ of cases laparotomy followed by repair of the bladder was carried out in 2 layers and the patients recovered uneventfully.

Perforation closure was done in 18 (12.1\%), cases of bowel injury. One patient of grade $\mathrm{V}$ hepatic injury was taken for damage control surgery but the patient was in cardiogenic shock and succumbed to death intraoperatively due to cardiorespiratory arrest in spite of prompt resuscitative measures.

The abdominal injuries were associated with various extra-abdominal injuries amongst which most common were rib fractures $88(28.8 \%)$ of cases [12].

Incidence of rib fracture was consistent with study conducted by Fazili et al. [13] but we accounted for higher amount of haemothorax and retroperitoneal hematomas.

The higher amount of rib fractures was probably due to increase number of upper abdominal trauma. Nonrecognition of an extra abdominal injury may contribute to the patients' death when a relatively simple procedure might otherwise have saved the patient's life.

Mortality rate in our study was $6(2 \%)$ of cases.

The major cause of mortality was delayed presentation of patients and poor general condition of patient. This was in contrast to studies conducted by Alli et al. [15] The reason for this was early presentation of patients in our study, early diagnosis and prompt surgical intervention.

The earliest presentation was at $30 \mathrm{~min}$ with one case presenting as late as 15 days after the injury. The early presentation of our patients helped us to start appropriate resuscitation at time and save many lives. Commonest postoperative complication in our study was wound infection $(12 \%)$ which in most cases were minor infections and were managed conservatively. This was consistent with studies conducted by Beall et al. [16]

The cause of sepsis/infection in these patients were necrotic tissue, mutilating injuries and late presentation in some patients. To conclude initial resuscitation measures and correct diagnosis forms the most vital part of blunt abdominal trauma management. Prompt evaluation of abdomen is mandatory to minimize preventable morbidity and mortality.

Early diagnosis can decrease mortality by $50 \%$.[18]

Mortality is related to delayed presentation and diagnosis, associated injuries and delayed surgical intervention. Clinical abdominal assessment is inaccurate of the BAT patients since there are often distracting injuries, altered levels of consciousness, non-specific signs and symptoms, and large differences in individual patient reactions to intraabdominal injury. Out of multiple modalities available for evaluating stable patients; CT scan along with StHd are best in evaluating which patient requires surgery or in deciding which patient can be safely discharged from emergency. The main drawbacks of CT scan are its cost, low sensitivity in detecting bowel injuries and hemodynamically unstable patients. Damage control laparotomy is a potentially life-saving procedure with the 
potential to mitigate the devastating clinical outcomes. [17, 19]

The swift recognition, timely and proper application of imaging methods in BAT patients along with physical examination have significantly decreased the number of non therapeutic and unnecessary laparotomies as a result and has increased NOM of solid organ injuries.

\section{Conclusions:}

The progress of patients with BAT has a close relationship with the number of injured organs. the number and presence of EAI organs were important which worsen patient conditions and influence at the prognosis. The time of hospitalization of patients has a lot to do with the result. early diagnosis and prompt treatment can save many lives

COI Statement: This paper has not been submitted in parallel. It has not been presented fully or partially at a meeting or podium or congress. It has not been published nor submitted for consideration beforehand.

This research received no specific grant from any funding agency in the public, commercial, or non-profit sectors. There are no relevant or minor financial relationships from authors, their relatives or next of kin with external companies.

Disclosure: The authors declared no conflict of interest. No funding was received for this study.

\section{References}

1. Van der Vlies CH, Olthof DC, Gaakeer M. Changing patterns in diagnostic strategies and the treatment of blunt injury to solid abdominal organs. Int J Emerg Med 2011; 4:47.

2. Agron Dogjani. (2013). Traumat e mbyllura të abdomenit, menaxhimi dhe trajtimi. In Traumat e mbyllura të abdomenit, menaxhimi dhe trajtimi (p. 282). "Botimet Barleti". https:// doi.org/10.5281/zenodo.5510592

3. Ahmet K, Tongue Y. Blunt abdominal trauma: evaluation of diagnostic options and surgical outcomes. Turkish J Trauma Emerg Surg 2008; 14: 205-10.

4. Marconi Fernandes T, Escocia Dorigatti A, Monteiro BT. Nonoperative management of splenic injury grade IV is safe using rigid protocol. Rev Col Bras Cir 2013;40: 323-8

5. DOGJANI,A., HAXHIREXHA, K., KOLANI, H., SELMANI, E., GJATA, A., TODAJ, M., TODAJ, A., \& BENDO, H. (2020). Correlation between Rib Fractures and Intraabdominal Organ Injuries after Blunt Chest Trauma. Albanian Journal of Trauma and Emergency Surgery, 4(2), 671-677. https://doi.org/10.32391/ajtes.v4i2.145
6. Hassan R, Aziz AA. Computerized tomography (CT) imaging of injuries of blunt abdominal trauma: a pictorial assay. Malays J Med Sci 2010; 17:29-39.

7. Taviloglu K, Yanar H. Current trends in the management of blunt solid organ injuries. Eur J Trauma Emerg Surg 2009; 35: 90-4.

8. Davis J, Cohn I, Nance F. Diagnosis and management of blunt abdominal trauma. Ann Surg 1996;183: 880-6.

9. Lowe RJ, Boyd DR, Frank CM, Baker RJ.The negative laparotomy for abdominal trauma. J Trauma 1997; 2:853-61.

10. Perry JF Jr, McCleelan RJ. Autopsy findings in 127 patients following fatal traffic accidents. Surg Gynaec Obstet 1964;119: 586-90.

11. Morton J, Hinshaw R. Blunt trauma to the abdomen. Ann Surg 1957; 145: 699-711.

12. Dogjani, A., Haxhirexha, K., Selmani, E., Bendo, H., Blloshmi, A., \& Craga, B. (2019). Considerations and Evaluations on Abdominal Trauma in Pediatric Age. Albanian Journal of Trauma and Emergency Surgery, 3(2), 424-438. https://doi.org/10.32391/ajtes.v3i2.64

13. Fazili A, Nazir S. Clinical profile and operative management of blunt abdominal trauma: a retrospective one-year experience at SMHS hospital, Kashmir, India. JK Practit 2001; 8: 219-21

14. Diercks DB, Mehrotra A, Nazarian DJ. Clinical policy: critical issues in the evaluation of adult patients presenting to the emergency department with acute blunt abdominal trauma. Ann Emerg Med 2011; 4: 57.

15. Alli N. Management of blunt abdominal trauma in Maiduguri: a retrospective study. Niger J Med 2005; 14: 17-22.

16. Beall AC, Bricker DL, Alessi FJ, et al. Surgical considerations in the management of civilian colon injuries. Ann Surg 1970; 173: 971-8.

17. Agron Dogjani. (2016). Damage Control Surgery, Procedura Kirurgjikale jetëshpëtuese. In Damage Control Surgery, Procedura Kirurgjikale jetëshpëtuese. (p. 165). "Botimet Barleti”. https://doi.org/10.5281/zenodo.5574575

18. Majid S, Gholamreza F, Mahmoud YM. New scoring system for intraabdominal injury diagnosis after blunt trauma. Chin J Traumatol 2014; 17: 19-24.

19. Wang S-Y, Liao C-H, Fu C-Y, Kang S-C. An outcome prediction model for exsanguinating patients with blunt abdominal trauma after damage control laparotomy: a retrospective study. BMC Surg 2014; 14: 24. 